\title{
Automatic segmentation of wrist bone fracture area by $K$-means pixel clustering from $X$-ray image
}

\author{
Kwang Baek Kim ${ }^{1}$, Doo Heon Song ${ }^{2}$, Sang-Seok Yun ${ }^{3}$ \\ ${ }^{1}$ Division of Computer Software Engineering, Silla University, Republic of Korea \\ ${ }^{2}$ Department of Computer Games, Yong-In SongDam College, Republic of Korea \\ ${ }^{3}$ Division of Mechanical Convergence Engineering, Silla University, Republic of Korea
}

\begin{tabular}{l} 
Article Info \\
\hline Article history: \\
Received Apr 20, 2019 \\
Revised Jul 9, 2019 \\
Accepted Jul 17, 2019 \\
\hline Keywords: \\
Fuzzy stretching \\
K-means \\
Pixel clustering \\
Wrist bone fracture \\
X-ray \\
\hline
\end{tabular}

\begin{abstract}
Early detection of subtle fracture is important particularly for the senior citizens' quality of life. Naked eye examination from X-ray image may cause false negatives due to operator subjectivity thus computer vision based automatic detection software is much needed in practice. In this paper, we propose an automatic extraction method for suspicious wrist fracture regions. We apply K-means in pixel clustering to form the candidate part of possible fracture from wrist X-ray image automatically. This method can recover previously detected patterned false cases with edge detection method after fuzzy stretching. The proposed method is successful in 16 out of 20 tested cases in experiment.
\end{abstract}

Copyright $(2019$ Institute of Advanced Engineering and Science. All rights reserved.

\section{Corresponding Author:}

Kwang Baek Kim,

Division of Computer Software Engineering,

Silla University,

Busan 46958, Republic of Korea.

Email: gbkim@silla.ac.kr

\section{INTRODUCTION}

Fracture refers to the complete or incomplete loss of continuity of bone, alveolar plate, or joint surface, often accompanied by damage to soft tissues or organs around the bone. The fracture of the forearm happens when at least one of the two important bones of the area between the elbow and the wrist - radius and ulna - is broken and frequently it accompanies with dislocation. It is caused by either indirect force in falling accident when the victim put one's hand on the floor with imbalance position or the direct force by traffic accident or contusion. Indirect force causes an oblique or spiral fracture, and the direct force causes a lateral fracture of the radial and ulna [1].

If the fracture is not treated properly so that the malunion or hyperkala bone occurs, the wrist is weakened with pain and the patient may feel difficulty in rotating hands [2]. The bone fracture detection is often through visual inspection of an X-ray image to determine the presence and severity of the fracture. The sources of difficulty in visual detection by radiologists might be the overlapping of several anatomical structures on an X-ray image or the inconsistency of image quality across exams. However, the potential human error from fatigue or operator subjectivity may increase the risk of missing subtle bone fractures [3]. Thus, automated bone fracture detection can assist doctors and paramedical staff to analyze the severity of the situation and to choose a proper course of action on urgent basis [4].

A typical automated bone fracture detection software consists of three stages-preprocessing, segmentation, and fracture detection. In this paper, we propose a computer vision based method to extract the candidate area of wrist fracture automatically from X-ray image. This step is the main source of automatic fracture detection and the preprocessing steps of this research is similar to our previous work [5]. 
There have been several researches in this automatic hand bone segmentation problems. Early attempt like [6] applies region based level set approach but no detailed experiment result was reported. In [7], it uses an extended version of Otsu's binarization but the computation time may take up several seconds. Many systems used some filtering algorithm and edge detection in their main part in segmentation [8-10] but none of them shows good experimental statistics in fracture detection rate. Some other work includes the operations like gray conversion, active contour segmentation but the detection rate was reported as low as $69 \%$ and still, it looks better than other simple method such as region growing and level set segmentation [6] for their implementation [11].

In this paper, we propose a pixel clustering based automatic segmentation of suspicious fractured area. This step should be done accurately before detecting the fractured spot. However, in many medical imaging applications, edge-based algorithms suffer because medical images commonly have poor contrasts, different types of noise, and missing or diffusive boundaries [12]. Instead, pixel clustering approaches have been successfully applied to detect the target organ from ultrasonography or X-ray images. Some examples of such approaches include detecting brain tumor [13, 14], brachial artery [15], cervical vertebrae [16], lung cancer [17], inflamed appendix [18], ganglion cyst [19] and breast image segmentation [20]. Thus, in this paper, we take K-means based pixel clustering approach $[16,21]$ to this automatic segmentation problem. Due to the low intensity contrast between the target area and the background, we need fuzzy stretching [22] to enhance the contrast.

\section{PIXEL CLUSTERING BY K-MEANS WITH FUZZY STRETCHING}

Image segmentation refers to the process of partitioning an image into mutually exclusive regions. It can be considered as the most essential and crucial process for facilitating the delineation, characterization, and visualization of regions of interest in any medical image [12]. Clustering can be defined as the optimal partitioning of a given set of $n$ data points into $c$ subgroups, such that data points belonging to the same group are as similar to each other as possible whereas data points from two different groups share the maximum difference. Image segmentation can be treated as a clustering problem where the features describing each pixel correspond to a pattern, and each image region corresponds to a cluster [23].

Very frequently, any vision based object extraction procedure provides auxiliary intensity stretching algorithm to extend the contrast to differentiate the target object from the background effectively [24]. From the given X-ray image, since the image does not have enough intensity contrast to discriminate bone part and other parts in the image, we have similar situation and thus adopt fuzzy stretching [22] for that purpose. In that fuzzy stretching, the dynamic control of fuzzy membership function for intensity stretching is the power of qualitative segmentation.

The grey-scale input image may not have enough brightness contrast between the "bright" side and the "dark" side. Thus, we stretch 0 's and 1's as follows so that the bright contrast is effectively exaggerated.

$$
X_{m}=\sum_{i=0}^{M \times N} X_{i} \times \frac{1}{M \times N}
$$

Let $X_{m}$ be the average brightness value of the image with $M \times N$ size, the distance from the brightest pixel and the darkest pixel are defined as (2).

$$
D_{\max }=\left|X_{h}-X_{m}\right|, \quad D_{\min }=\left|X_{m}-X_{l}\right|
$$

The brightness adjustment value is computed as shown in (3).

$$
\begin{aligned}
& \text { if }\left(X_{m}>128\right) \text { adjustment }=255-X_{m} \\
& \text { elseif }\left(X_{m} \leq D_{\min }\right) \text { adjustment }=D_{\min } \\
& \text { elseif }\left(X_{m} \geq D_{\max }\right) \text { adjustment }=D_{\max } \\
& \text { else adjustment }=X_{m}
\end{aligned}
$$

Thus, the maximum, minimum, and the center point of the brightness which will form the fuzzy membership triangle are defined as follows;

$$
I_{\max }=X_{m}+\text { adjustment, } I_{\min }=X_{m} \text {-adjustment }
$$




$$
I_{\text {mid }}=\frac{I_{\max }+I_{\min }}{2}
$$

The membership function is then defined as Figure 1.

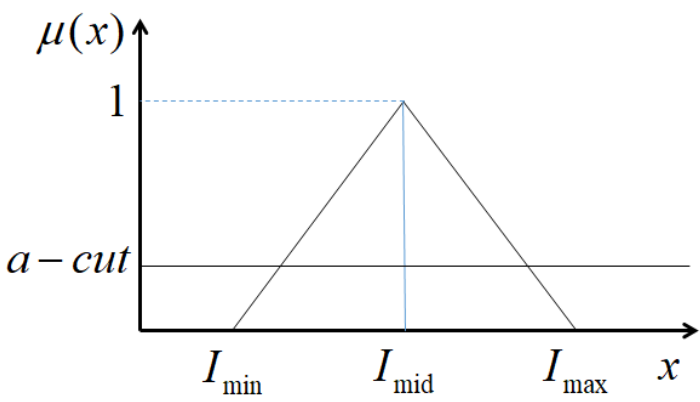

Figure 1. Fuzzy membership function for brightness enhancement

The cut point ( $\alpha-c u t$ ) in Figure 2 is computed as follows;

$$
\begin{aligned}
& \text { if }\left(I_{\min }>0\right) \alpha-c u t=I_{\min } / I_{\max } \\
& \text { else } \alpha-c u t=0.5 \\
& X_{\text {new }}=255 \times \frac{X-\alpha}{\beta-\alpha}
\end{aligned}
$$

The upper limit value ( $\alpha$-cut) and the lower limit value $(\alpha)$ are defined as the highest and lowest $X_{i}$ among pixels that have higher membership degree than the cut point $\alpha$-cut. Then, the final stretching equation for the pixel brightness is defined as follows;

After such fuzzy stretching, within the range of target object existence, we try to apply pixel clustering. K-means clustering is a well-known unsupervised learning algorithm that has many variants with respect to the application and we follow a speed up version of it [20] as summarized in Algorithm 1.

Algorithm 1. K-means process: a speedup version

Begin Initialize $n, k, \mu_{1}, \mu_{2}, \ldots, \mu_{k}$

Do classify $n$ samples according to nearest $\mu_{i}$

recompute $\mu_{i}$

Until no change in $\mu_{i}$

Return $\mu_{1}, \mu_{2}, \ldots, \mu_{k}$

End

The principle of K-means is as simple as above description. From arbitrarily given $k$ sets, the algorithm iteratively re-assigns the data sets according to the center point and re-computes center points at every iteration until there is no significant change. The typical result of K-means pixel clustering is shown as Figure 2.

From the K-means clustering result as shown in Figure 3(a), we need noise removal process based on the domain knowledge such that the fractured area does not have extreme intensity values and then we apply labelling procedure [25] to form and remove the contour of the wrist bone as shown in Figure 3(b). Then the region labeling procedure is applied to make the target suspicious fracture repeatedly such that the suspicious regions are connected and form a meaningful size of object while removing small or disconnected noises as shown in Figure 3(c). That completes our automatic segmentation process. 

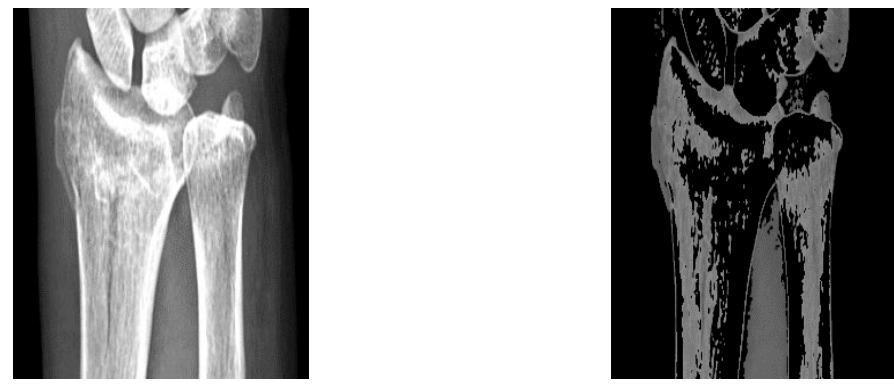

Figure 2. Candidate fractured area extraction by K-means,

(a) Image after fuzzy stretching, (b) Candidate fractured area after K-means
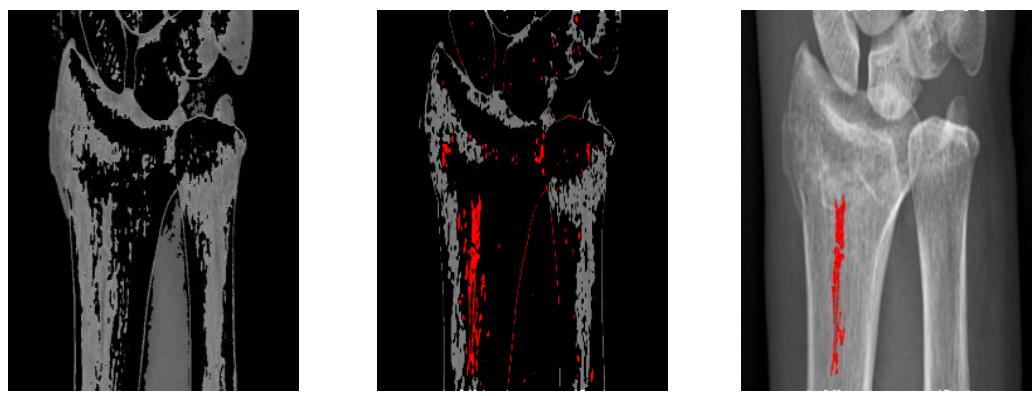

Figure 3. Fracture area extraction after k-means,

(a) After K-means, (b) Candidate fracture, (c) Fracture area

\section{EXPERIMENT}

The proposed method is implemented in C\# under Visual Studio 2017 environment with Intel(R) Dual Core(TM) i3-5005U CPU @ 2.0 GHz and 4 GB RAM PC. Twenty (20) X-Ray images containing hand bone skeleton with fracture in some place of $1208 \times 1502$ size were used in this experiment. In experiment, our cooperated pathologist evaluate if the result come out of the software is sufficiently meaningful. The pathologist agrees with the proposed method's results in 16 out of 20 cases. Figure 4 demonstrates both the successful extraction case (Figure 4(a)) and failed extraction (Figure 4(b)).
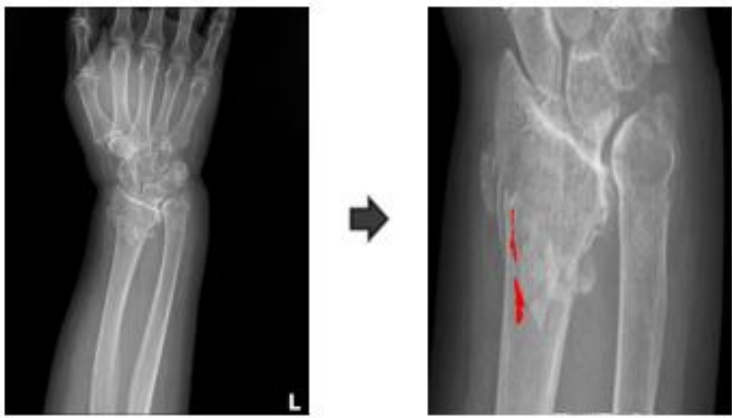

(a)
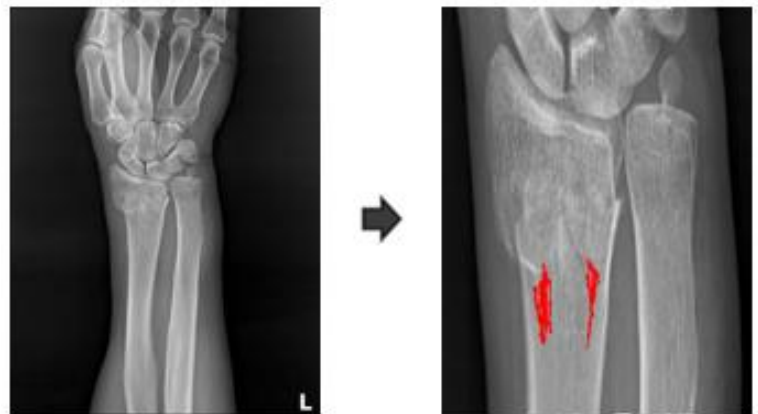

(b)

Figure 4. Fracture area extraction success and failed cases, (a) Successful extraction (b) Failed extraction

In retrospective analysis, failed extraction cases represent either insufficient discrimination of fractured area from normal state in intensity or unintended removal of small but meaningful fractured part in region labelling process. The former case needs careful filming or better intensity stretching procedure in the future. For the latter case, it is partly attributed to the intrinsic disadvantage of K-means that it needs prior determination of the number of clusters before processing. That is, the algorithm requires a good initialization from the experience of the environment characteristics. 


\section{CONCLUSION}

In this paper, we propose a method that extracts the suspicious fractured area sutomatically from $\mathrm{X}$-ray images. Such automatic segmentation is crucial to build a highly tibust and accurate fracture detection software. Like many other prior studies, our previous study [5] used canny edge algorithm to take the fracture part around the wrist area. However, the result shows that there were a patterned failure in detecting the target fracture location. Thus, we take K-means pixel clustering as the answer of such problem.

While this pixel clustering approach is more robust than edge detection schemes, it still needs to be improved due to its static initialization problem. In experiment, the proposed method exhibits a respectable but improvable successful extraction rate (human pathologist agrees with the software (16 out of 20 cases or $80 \%$ accurate based on the human expert's decision as the ground truth). We need more careful intensity stretching method and more stable pixel clystering algortithms in order to develop better automatic fracture detection software.

\section{ACKNOWLEDGMENT}

This work was supported by the Technology Innovation Program (20000515, IoT based Assistive Robot Systems for Personalized Healthcare) funded By the Ministry of Trade, Industry \& Energy (MOTIE, Korea).

\section{REFERENCES}

[1] K. Okada, Visual Navi Orthopedics, (Lee, HS, Kim, TG. Transl.) Seoul, Hansole Medical Books. 2014.

[2] S. Y. Park, et al., "Surgical or nonsurgical treatment of osteoporotic fractures," J Korean Med Assoc, vol. 59, no. 11, pp. 857-865, 2016.

[3] Y. Cao, et al., "Fracture detection in x-ray images through stacked random forests feature fusion," In Proc. of Biomedical Imaging, 2015 IEEE 12th International Symposium on IEEE, N.Y., USA, pp. 801-805, 2015.

[4] O. Bandyopadhyay, et al., "Long-bone fracture detection in digital X-ray images based on digital-geometric techniques," Computer methods and programs in biomedicine, vol. 123, pp. 2-14, 2016.

[5] K. B. Kim and D. H. Song, "Automatic Hand Bone Segmentation with Fuzzy Stretching from X-ray Image," Far East Journal of Electronics and Communications, vol. 18, no. 1, pp. 147-157, 2018.

[6] Lin, P, et al., "X-ray carpal-bone image boundary feature analysis using region statistical feature based level set method for skeletal age assessment application," Optica Applicata, vol. 35, no. 2, pp. 283-294, 2005.

[7] A. A. Tirodkar, "A multi-stage algorithm for enhanced x-ray image segmentation," International Journal of Engineering Science Technology (IJESE), vol. 3, no. 9, pp. 7056-7065, 2011.

[8] S. Hao, et al., "Automatic Isolation of Carpal-Bone in Hand X-Ray Medical Image," In: Du W. Editor Informatics and Management Science I. Lecture Notes in Electrical Engineering, London, Springer. vol. 204, pp. 657-662, 2013.

[9] M. Al-Ayyoub, et al., "Detecting Hand Bone Fractures in X-Ray Images," Journal of Multimedia Processing and Technologies, vol. 4, no. 3, pp. 155-168, 2013.

[10] A. K. O. Sudana and S. Kom, "Bone Fracture Detection using OpenCV," Journal of Theoretical and Applied Information Technology, vol. 64, pp. 249-254, 2014.

[11] M. U. Joshi and S. T. Gandhe, "Bone fracture detection using active contour segmentation," International Journal of Applied Engineering Research, vol. 11, no. 6, pp. 4230-4234, 2016.

[12] A. Chien, et al., "Frame-based segmentation for medical images," Communications in Mathematical Sciences, vol. 9, no. 2, pp. 551-559, 2011.

[13] E. Abdel-Maksoud, et al., "Brain tumor segmentation based on a hybrid clustering technique," Egyptian Informatics Journal, vol. 16, no. 1, pp. 71-81, 2015.

[14] V. P. Ananthi, et al., "A new fuzzy clustering algorithm for the segmentation of brain tumor," Soft Computing, vol. 20, no. 12, pp. 4859-4879, 2016.

[15] J. Park, et al., "Automatic Segmentation of Brachial Artery based on Fuzzy C-Means Pixel Clustering from Ultrasound Images," International Journal of Electrical and Computer Engineering (IJECE), vol. 8, no. 2, pp. 638-643, 2018.

[16] H. J. Lee, et al., "Effective Computer-Assisted Automatic Cervical Vertebrae Extraction with Rehabilitative Ultrasound Imaging by using K-means Clustering," International Journal of Electrical and Computer Engineering (IJECE), vol. 6, no. 6, pp. 2810-2817, 2016.

[17] M. A. Hussain, et al., "Lung cancer detection using artificial neural network \& fuzzy clustering," International Journal of Advanced Research in Computer and Communication Engineering, vol. 4, no. 3, pp. 360-363, 2015.

[18] K. B. Kim, et al., "Automatic Extraction of Appendix from Ultrasonography with Self-Organizing Map and ShapeBrightness Pattern Learning," BioMed research international, 2016.

[19] A. Suryadibrata and K. B. Kim, "Ganglion Cyst Region Extraction from Ultrasound Images Using Possibilistic C-Means Clustering Method," Journal of information and communication convergence engineering, vol. 5, no. 1, pp. 49-52, 2017.

[20] H. M. Moftah, et al., "Adaptive k-means clustering algorithm for MR breast image segmentation," Neural Computing and Applications, vol. 24, no. 7-8, pp. 1917-1928, 2014. 
[21] T. H. Sarma, et al., "A hybrid approach to speed-up the k-means clustering method," International Journal of Machine Learning and Cybernetics, vol. 4, no. 2, pp. 107-117, 2013.

[22] K. B. Kim and D. H. Song, "Defect detection method using fuzzy stretching and ART2 learning from ceramic images," International Journal of Software Engineering and Its Applications, vol. 8, no. 9, pp. 29-38, 2014.

[23] A. K. Jain, et al., "Data clustering: a review," ACM computing surveys, vol. 31, no. 3, pp. 264-323, 1999.

[24] K. B. Kim and D. H. Song, "Automatic Defect Inspection with Adaptive Binarization and Bresenham's algorithm for Spectacle Lens Products," Journal of the Korea Institute of Information and Communication Engineering, vol. 21, no. 7, pp. 1429-1434, 2017.

[25] R. C. Gonzales and R. E. Woods, Digital Image processing (3td Ed.), Prentice Hall, NJ, USA, 2007.

\section{BIOGRAPHIES OF AUTHORS}
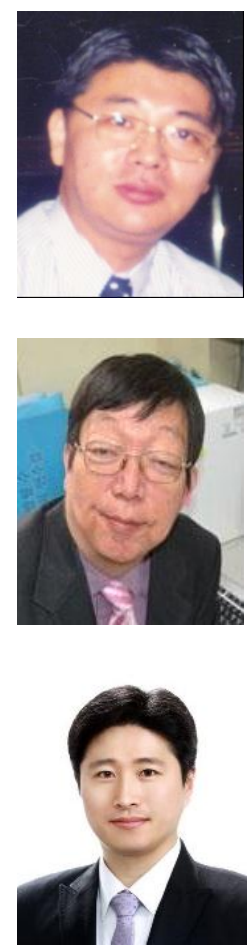

Kwang Baek Kim received his M.S. and Ph.D. degrees from the Department of Computer Science, Pusan National University, Busan, Korea, in 1993 and 1999, respectively. From 1997 to the present, he is a professor at the Division of Computer and Information Engineering, Silla University, Korea. He is currently an associate editor for Journal of Intelligence and Information Systems and The Journal of Information and Communication Convergence Engineering. His research interests include machine learning, fuzzy clustering and fuzzy control system, data mining, image processing, and bioinformatics.

Doo Heon Song received his B.S. from Seoul National University in 1981 and M.S. from the Korea Advanced Institute of Science and Technology in 1983 in Computer Science. $\mathrm{He}$ received his Ph.D. Certificate in Computer Science from the University of California at Irvine in 1994. He has been a professor at Department of Computer Games, Yong-in Songdam College, Korea, since 1997. His research interests include artificial intelligence, fuzzy systems, image processing, and computer game design. He is currently the associate editor of Journal of Information and Comunication Convergence Engineeing.

Sang-Seok Yun received the B.S. in Mechanical Engineering from Inje University in 2002, and his M.S. in Mechatronics Engineering from Gwangju Institute of Science and Technology (GIST), Korea, in 2005. He received his Ph.D. in Mechanical Engineering from Korea University in 2013. He was a researcher at the Korea Institute of Science and Technology (KIST) from 2005 to 2017. He is currently an assistant professor at the Division of Mechanical Convergence Engineering, Silla University, Korea. His current research interests include cognitive control system, human-robot interaction, and socially assistive robots. 